\title{
Tele Education in Diabetic Patients during Coronavirus Outbreak
}

\author{
Kamran Dehghan ${ }^{1}$, Morad Ali Zareipour ${ }^{2 *}$, Sahar Zamaniahari ${ }^{2}$, Mina Tasouji Azari $^{3}$ \\ ${ }^{1}$ Department of Pediatric Disease, School of Medicine Shahid Motahari Hospital Urmia University of Medical Sciences, Urmia, \\ Iran; ${ }^{2}$ Health System Research Unit, Health Center of Urmia, Urmia University of Medical Sciences, Urmia, Iran; ${ }^{3}$ Department \\ of English Language, Islamic Azad University, Tabriz, Iran
}

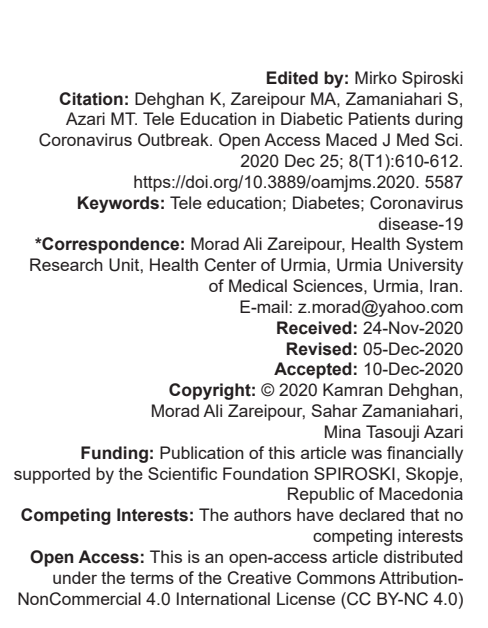

Abstract

The number of cases of infection to coronavirus disease (COVID)-19, human casualties and economic losses are increasing, and one of the high-risk groups in which the risk of infection and death is high is diabetics. People with diabetes experience severe symptoms and complications if they are infected by COVID-19. One way to control and prevent the spread of the coronavirus is to stay home, avoid daily physical interactions, and quarantine at home. Due to the high prevalence of diabetes and the importance of long-term follow-up, Tele education in diabetic patients is one of the methods that provide care using communication means. As the quality of information received by diabetic patients improves, their awareness of treatment goals and, consequently, their acceptance of treatment methods will increase, which in turn will lead to greater effectiveness and better treatment outcomes. Finally, Tele education reduces contact between diabetics and physicians, therapists, and health care providers, thereby reducing the risk of disease.

\section{Statement of the Problem}

World Health Organization announced coronavirus disease (COVID)-19 as an epidemic, a new illness which is different from other viruses such as severe acute respiratory syndrome, Middle East Respiratory Syndrome, and influenza [1]. The number of cases of infection to COVID-19 and its human casualties are increasing worldwide; along with the economic losses resulting from the illness is much higher, estimated to go beyond the capacity of the developing and developed countries in coming months [2]. One of the high-risk groups in which the risk of infection and death is high is diabetics, in which the mortality rate out of COVID-19 is reported to be $\% 9 / 2$ among diabetics while it is $\% 1 / 4$ for normal healthy people [3]. People with diabetes experience severe symptoms and complications if they are infected by COVID-19. If the diabetes is controlled properly, the risk of experiencing acute symptoms of COVID-1 infection would be the same as common people, while if it is not controlled along with fluctuation in blood sugar, the person would encounter the complications and difficulties resulting from diabetes. As other infectious illnesses, heart disease as well as other disease together with the diabetes increases the risk of illness severity of COVID-19. Infectious diseases also cause the increase in internal inflation, because the ability of the body is reduced in fighting against infection. The reason of this complication is the high rate of blood sugar leading to incidence of more severe problems and complications [4]. The need to self-care and self-control to prevent and control the spread of the coronavirus is of vital importance. One way to control and prevent the spread of the coronavirus is to stay home, avoid daily physical interactions, and quarantine at home [1]. The crisis caused by this virus affects the patients' education system of all countries including Iran, leading to shut down of attendance courses of the patients [5]. In addition to education programs, it seems necessary to protect patients suffering from diabetes who have self-care problems with the administration of the follow-up program with the purpose of promoting the awareness, as well as improving the performance and attitudes. Although it is possible to follow the treatment through regular in-person attendance of the patient or visiting at home, there should be other low-cost and applicable methods for a large number of the patients due to the highly growing spread of the diabetes and the importance of its long-term follow-up. Today, Tele education makes the health-care provider able to evaluate, educate, collect data, intervene, and protect 
the patient's family [6]. Among the media used in Tele education, phone is regarded as the highly accessible tool for the majority of people.

\section{Tele Education}

One of the implications of tele medical services is tele education. It is one of the ways that provides health care using some media as videos, Internet, and phone. Throughout this technique, health care providers communicate with the patients or the medical centers using camera, video conferencing, phone and videos, attempting to educate and advise [7]. This technology leads to quick access of the individual to the health advisory services, reduction in cost, and accessibility to the most appropriate specialized skills, as well as an inclusive improvement in the quality of life. In addition, it makes people to attend virtually, instead of in person attendance over the long distance, so it would pass a great mass of information in short time from far distance, therefore, reduced the expenses [8].

\section{Tele Education for the Diabetics}

Education is one of the most important aspects of health service providing. Patients are highly interested in receiving information on their hearth status. When the quality of the received information by the diabetic patient increases, his/her awareness on the therapeutic goals, and consecutively, the acceptance of the treatment intervention would increase, leading to more effectiveness and better therapeutic results. Reaching to higher level of patient satisfaction is another achievement of patient education. COVID-19 pandemic not only put the health care providers under great pressure but also strongly changed the way of patients' education and their access to the hearth care services. COVID-19 pandemic highlights the importance of tele-education to prevent the infection caused by direct contact. Without any vaccine or effective treatment, the only satisfactory substitutes for in-person hearth care services are quarantine and observance of social distances. Tele education is an attractive, efficient, and affordable option in fighting against coronavirus for patients suffering from the diabetes.

Tele education for the diabetics in corona outbreak can de classified into two groups: (1) Diabetes self-care and (2) COVID-19 self-care.

Some instances of diabetes self-care tele education are education on consuming low volume meals more times, observing the program of health dish, consuming foods with low glycemic index such as vegetables and whole grains, drinking 6-8 glasses of liquids daily, using two to three units of fruits daily, avoiding too much use of fried and fast foods and sweets, consuming dairies, especially probiotics and enriched ones with Vitamin D daily, using low-fat proteins such as chicken, fish, meat, beans, and eggs. Dietary carbohydrate restriction, because it reliably reduces high blood glucose, does not require weight loss (although is still best for weight loss), and leads to the reduction or elimination of medication. On the other hand, since obesity is a highly prevalent comorbidity in severe cases of COVID-19, a decrease in body mass index has a significant effect on reducing the risk of developing COVID-19 disease or reducing the severity of symptoms in case of infection. All documents reported that there are currently no known supplements to prevent COVID-19 so we emphasize that there is no need to avoid different supplements [9].

It is recommended that diabetic patients have a hemoglobin A1C (HbA1C) test, 3-4 times a year and then consult with their doctor about the treatment process. $\mathrm{HbA} 1 \mathrm{C}<7 \%$ indicates diabetes control and the values above $8 \%$ indicate that the patient should reconsider the treatment of diabetes. Therefore, the goal of successful diabetes treatment is to reduce the $\mathrm{HbA} 1 \mathrm{C}$ level to $<7 \%$ [10].

Another way to control your blood sugar is to exercise. Exercise for diabetics will cause glucose and glycogen to be burned and consumed within the muscles. During and after exercise, blood sugar enters the muscles and glucose levels are regulated in the body. In this way, exercise helps diabetics treat and control their diabetes [9]. Thus, doing light aerobics exercises weekly and keeping the optimal weight, taking the diabetes medicines regularly, doing blood sugar checkup test at home, keeping the optimal blood sugar, asking one of young and healthy family members' help for medicine prescriptions, home visiting and tele home care if possible, following the instructions of the doctor, regular contact with the doctor, regular examination of the health status of the feet, avoiding smoking, and controlling stress, anxiety, and tension.

Some instances of tele education on COVID-19 self-care are as follows: education on avoiding to contact with people suffering from symptoms similar to coronavirus and influenza, wearing masks while going out, avoiding to kiss or shake hand, keeping the social distance (at least one meter and a half), washing hands regularly with soap for $20 \mathrm{~s}$, using alcohol disinfectants for hands out of home, avoiding to touch the public utilities and surfaces in crowded places and public means of transportation, avoiding to touch eyes and face, especially out of home, avoiding big parties and mass gatherings, and staying at home as much as possible.

Regarding the above-mentioned tips as well as the present condition of the country encountering COVID-19 illness, implementing Tele education for diabetic patients would be significantly beneficial in following ways: 


\section{Reducing treatment expenses with a References} decrease in symptoms and preventing sever complications

2. Preventing the consequences and severity of the illness

3. Improving the quality of the care and enabling the patient and family in their self-care

4. Reducing the time of hospitalization and the rate of re-hospitalization of the patient

5. Increasing the patient's observance of the treatment procedure and needed follow-ups

6. Assisting the patient and his/her family in gaining independence and self-sufficiency (self-management and self-care)

7. Reducing stress, anxiety, and mental problems

8. Improving the patients' quality of living

9. Increasing the satisfaction of the patient and his/her family

10. Reducing the unnecessary contact of the diabetic patients with doctors, therapists, and health care providers, leading to the decrease in encountering the illness

11. Improving the awareness level of the diabetic patients of their body and soul health, using the active participation of the patient in his/her own health control.

\section{Conclusion}

Tele education can be influential in prevention and control COVID-19 illness. The education which is performed with peace, concentration effortlessly can result in significant learning. Since tele education is done at home, it would be possible for diabetic patients to control their illness with more concentration and peace. Obviously, there are some inhibiting factors that prevent the diabetic patients from benefiting from this type of education properly. However, tele education can be influential for the diabetic patients more likely to encounter COVID-19, because of being attractive, efficient, and influential.

1. Hou C, Chen J, Zhou Y, Hua L, Yuan J, He S, et al. The effectiveness of quarantine of Wuhan city against the Corona virus disease 2019 (COVID-19): A well-mixed SEIR model analysis. J Med Virol. 2020;92(7):841-8. https://doi.org/10.1002/ jmv.25827

PMid:32243599

2. Zareipour M, Kalejahi JN. The role of social participation in controlling and preventing of Coronavirus 2019 disease in Iran. Open Access Maced J Med Sci. 2020;8(T1):1-3. https://doi. org/10.3889/oamjms.2020.4956

3. Zareipour M, Movahed E, Jadgal MS, Zamaniahari S. Role of self-care in diabetic patients during COVID-19 pandemic. J Diabetes Nurs. 2020;8(3):1131-6.

4. Yang J, Zheng Y, Gou X, Pu K, Chen Z, Guo Q, et al. Prevalence of comorbidities and its effects in patients infected with SARSCoV-2: A systematic review and meta-analysis. Int J Infect Dis. 2020;94:91-5. https://doi.org/10.1016/j.ijid.2020.03.017

PMid:32173574

5. Ahmady S, Shahbazi S, Heidari M. Transition to virtual learning during Coronavirus Disease-2019 crisis in Iran: Opportunity or challenge. Disaster Med Public Health Prep. 2020;14(3):e11-2. https://doi.org/10.1017/dmp.2020.142

PMid:32375914

6. Peck A. Changing the face of standard nursing practice through telehealth and telenursing. Nurs Adm Q. 2005;29(4):339-43. https://doi.org/10.1097/00006216-200510000-00008

PMid: 16260998

7. Black JM, Hawks JH. Medical-Surgical Nursing. Amsterdam: Elsevier, Saunders; 2005.

8. Holmqvist M, Vincent N, Walsh K. Web-vs. telehealth-based delivery of cognitive behavioral therapy for insomnia: A randomized controlled trial. Sleep Med. 2014;15(2):187-95. https://doi.org/10.1016/j.sleep.2013.10.013

PMid:24461370

9. Gao F, Zheng KI, Wang XB, Sun QF, Pan KH, Wang TY, et al. Obesity is a risk factor for greater COVID-19 severity. Diabetes Care. 2020;43(7):e72-4. https://doi.org/10.2337/dc20-0682 PMid:32409499

10. Feinman RD, Pogozelski WK, Astrup A, Bernstein RK, Fine EJ, Westman EC, et al. Dietary carbohydrate restriction as the first approach in diabetes management: Critical review and evidence base. Nutrition. 2015;31(1):1-13.

PMid:25287761 